\title{
Multilinguales
}

\section{L'approche par les compétences en Algérie : de la théorie à la pratique}

The skills approach in Algeria : from theory to practice

\section{M'hand Ammouden}

\section{OpenEdition}

Journals

Édition électronique

URL : https://journals.openedition.org/multilinguales/3733

DOI : $10.4000 /$ multilinguales.3733

ISSN : 2335-1853

Éditeur

Université Abderrahmane Mira - Bejaia

Référence électronique

M'hand Ammouden, «L'approche par les compétences en Algérie : de la théorie à la pratique »,

Multilinguales [En ligne], 10 | 2018, mis en ligne le 31 décembre 2018, consulté le 21 septembre 2021.

URL : http://journals.openedition.org/multilinguales/3733 ; DOI : https://doi.org/10.4000/

multilinguales.3733

Ce document a été généré automatiquement le 21 septembre 2021.

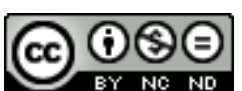

Multilinguales est mise à disposition selon les termes de la Licence Creative Commons Attribution Pas d'Utilisation Commerciale - Pas de Modification 4.0 International 


\title{
L'approche par les compétences en Algérie : de la théorie à la pratique
}

The skills approach in Algeria : from theory to practice

\author{
M'hand Ammouden
}

1 L'Algérie a décidé d'amorcer, il y a presque deux décennies, une réforme globale et progressive de son système éducatif. Cela s'est, entre autres, traduit par la recommandation d'adopter l'Approche Par les Compétences (désormais APC) dans les trois paliers de son système : le Primaire, le Moyen et le Secondaire. Dans quelle mesure les principaux fondements de cette approche sont-ils pris en considération par les auteurs des documents théoriques du Ministère de l'Éducation Nationale (désormais MEN) et par les concepteurs des programmes et des manuels scolaires de langue française? Qu'en pensent les enseignants? Ont-ils adopté les changements « décrétés »?

2 Pour apporter des éléments de réponses à ces questions, nous commençons par rappeler quelques-uns des principaux fondements théoriques de cette approche. Nous citons ensuite certains des principes mis en avant dans les documents théoriques émanant du MEN, dont le Référentiel général des programmes (Désormais RGP) et le Guide méthodologique d'élaboration des programmes (Désormais GMEP), conçus en 2009 par la Commission Nationale des Programmes. Nous consacrons enfin l'essentiel de l'article à l'examen du degré de conformité de l'enseignement/apprentissage actuel du français à à une dizaine des plus importants fondements de l'APC.

3 Nous nous appuyons essentiellement sur l'examen de plusieurs manuels, programmes et documents d'accompagnement émanant du ministère et sur les résultats d'un questionnaire remplis par 322 enseignants de français, représentatifs d'une quarantaine de wilayas. Il s'agit de 116 PEM (Professeur de l'Enseignement Primaire), de 109 PEM (du Moyen) et de 102 PES (du Lycée). Le questionnaire a été distribué au cours de l'année scolaire 2017-2018. 


\section{Qu'est-ce que l'approche par les compétences?}

4 L'APC apparait au début des années 1990. C'est une approche centrée sur l'apprenant, mais aussi et surtout sur l'activité de celui-ci et donc sur l'apprentissage (actif). Cette approche s'inspire du mouvement de l'École Nouvelle du début du siècle précédent, s'inscrit notamment dans le courant socioconstructiviste (Boutin, 2005) et fait ainsi partie des modèles qui «attribuent à l'apprenant un rôle déterminant dans l'édification de ses compétences, de ses savoirs et de ses connaissances » (Carbonneau et Legendre, 2002).

On prône d'abandonner la logique de la transmission des connaissances encyclopédiques, par l'enseignant à un apprenant passif, au profit de celle de l'apprentissage qui s'appuie davantage sur l'activité des apprenants. On rejette aussi les fondements behavioristes de la pédagogie par objectifs (PPO) au profit du cognitivisme (Ibid.). On insiste également souvent sur l'importance de favoriser le transfert des apprentissages en dehors de l'école (Ait Djida, 2009; Ammouden, 2017 ; Perrenoud, 2008). Dans la présentation du livre consacré par Perrenoud (2008) à cette approche, il est expliqué que

Réussir à l'école n'est pas une fin en soi. (...) L'élève devrait être capable de mobiliser ses acquis scolaires en dehors de l'école, dans des situations diverses, complexes, imprévisibles. Aujourd'hui, cette préoccupation s'exprime dans ce qu'on appelle assez souvent la problématique du transfert des connaissances ou de la construction de compétences.

6 Ces principes exigent le recours à des démarches et dispositifs didactiques, tels que les projet-élèves (Huber, 2005), qui favorisent l'interdisciplinarité, le développement des compétences de haut niveau et le transfert: l'APC implique l'apprentissage par situations-problèmes "dans le cadre d'une pédagogie du projet" et exige que les apprenants soient "actifs et engagés dans leurs apprentissages " (Perrenoud, 1995). Nous pouvons ainsi considérer, à la suite de Beacco (2007), que l'APC constitue une passerelle entre les approches communicatives et l'approche actionnelle.

7 L'APC implique enfin l'allègement des programmes (Perrenoud, 2008), parce que le développement des compétences exige plus de temps que la transmission des connaissances.

\section{Les choix théoriques institutionnels?}

8 En définissant l'APC, les auteurs du RGP expliquent que cette approche privilégie « une logique d'apprentissage centrée sur l'élève, sur ses actions et réactions face à des situations-problèmes » aux dépens de celle de l'enseignement " basée sur les savoirs et sur les connaissances à faire acquérir ». L'apprenant doit alors être entrainé à " agir (chercher l'information, organiser, analyser des situations, élaborer des hypothèses, évaluer des solutions, ...) » par des situations-problèmes représentant « des situations de vie susceptibles de se présenter à lui avec une certaine fréquence ». (RGP, $2009: 21$ ).

En expliquant les implications de la réforme sur le plan pédagogique, les concepteurs du GMEP (2009:26-27) retiennent d'abord le courant socioconstructiviste. En traitant ensuite de l'APC et de ses principales caractéristiques, ils notent que ce qui fonde la compétence "c'est la situation complexe qui lui permet de se révéler; il faut que l'apprenant heurte un obstacle suffisamment important pour provoquer la mobilisation de ses connaissances ». Ils ajoutent que « les obstacles artificiels situés en dehors de la 
zone développement proximale ne sont pas motivants, et ne permettent pas le développement de compétences ", avant de citer Perrenoud (cité par GMEP, 2009 : 26) qui affirme qu' "on ne construit des compétences qu'en affrontant de vrais obstacles dans une démarche de projets ou de résolution de problèmes ».

On explique par ailleurs dans plusieurs documents destinés aux enseignants que les programmes doivent être formulés en termes de compétences; et qu'une compétence consiste à mobiliser des savoirs, des savoir-faire et des attitudes permettant « d'identifier et d'accomplir un certain nombre de tâches appartenant à une famille de situations, que ces dernières soient disciplinaires ou transversales ». Le projet est donc « le moyen d'apprentissage adéquat » dans l'APC.

11 On insiste ainsi beaucoup sur l'importance de travailler par le dispositif du «projet pédagogique» qui vise des objectifs transversaux "qui se réfêrent à l'ensemble des disciplines", ce qui implique que les différents acteurs de l'école aient "une certaine marge de manouvre, d'initiative et de liberté » et que la rigidité laisse " place à une certaine souplesse pour permettre de donner en priorité du sens aux activités que l'on veut mettre en œuvre après une clarification des objectifs et l'établissement des échéanciers ». Il faudrait retenir des compétences précises en tenant compte, entre autres, des profils des apprenants et «des difficultés et contraintes de différentes natures (matérielles, sociales, éthiques...) » (RGP, 2009 :29).

12 Les auteurs du GMEP (2009 : 30) expliquent, quant à eux, que la démarche du projet pédagogique :

- est une entreprise collective gérée par le groupe-classe où l'enseignant anime, mais ne décide pas de tout ;

- s'oriente vers une production concrète (au sens large : texte, journal, exposition, maquette, carte, expérience scientifique, création artistique ou artisanale, enquête, sortie, concours, jeu, etc.) ;

- induit un ensemble de tâches dans lesquelles tous les élèves peuvent s'impliquer et jouer un rôle actif, qui peut varier en fonction de leurs moyens et intérêts ;

- suscite l'apprentissage de savoirs et de savoir-faire de gestion de projet (mobiliser les ressources, décider, planifier, coordonner, etc.) ;

- favorise en même temps des apprentissages identifiables (au moins après-coup) figurant au programme d'une ou plusieurs disciplines. (2009: 30).

On traite, enfin, souvent des situations d'intégration et des situations-problèmes. Les premières des situations d'apprentissage ou d'évaluation complexes qui se présentent généralement sous la forme d'une situation-problème

ayant pour but l'intégration et la mobilisation des acquis (valeurs, compétences transversales et disciplinaires, savoirs déclaratifs, procéduraux et conditionnels, attitudes, conduites et comportements) et visant à réaliser une compétence globale qui couronne un cursus plus ou moins étalé dans le temps : cycle, palier et année. (GMEP, 2009 : 28)

14 La situation-problème doit être concrète, avoir du sens pour l'élève, l'interpeller et susciter en lui un questionnement. Fondée sur l'activité de l'apprenant, elle lui donne l'occasion "d'expliquer sa démarche, d'expliciter sa pensée et de justifier ses choix pour répondre aux questions posées ou au problème à résoudre » (GMEP, 2009 : 28)

15 Ces orientations et définitions correspondent très bien à celles qu'on trouve dans les écrits des théoriciens de l'APC. Qu'en est-il du degré de prise en compte de ces principes dans les programmes, les manuels, les pratiques des enseignants? 


\section{La centration sur l'apprenant?}

\section{elles prises en considération?}

La majorité de nos enquêtés pense que les programmes correspondent peu (58\%) ou pas du tout ( $20 \%)$ aux besoins des apprenants. La moitié de ceux qui répondent par «oui » sont des PEP et la majorité de ceux qui disent «non » sont des PES ; tandis que $59 \%$ des PEM optent pour la réponse "un peu». Nous concluons que, selon les réponses des enseignants, au fur et à mesure qu'on s'éloigne du Primaire, les programmes s'écartent des besoins des apprenants.

Qu'en est-il des contenus des manuels ? Ils correspondraient encore moins que ceux des programmes aux besoins des apprenants selon les enseignants : «oui » $(18 \%)$, « un peu » $(45 \%)$, «non » $(37 \%)$.

Ces contenus motivent-ils les apprenants ? $14 \%$ seulement répondent par « Oui ». Les autres optent pour la réponse « un peu » $(50 \%)$ ou « non » $(36 \%)$.

En outre, si $78 \%$ de nos enquêtés pensent que les productions visées par les projetsélèves sont peu $(53 \%)$ ou pas du tout motivantes $(25 \%)$, le pourcentage des PES qui répondent par " oui » diminue à $18 \%$, tandis que taux de ceux qui optent pour «non » monte à $33 \%$.

Les enseignants pensent par exemple que :

$\mathrm{P}^{1} 135$. Le programme ne répond pas aux besoins des élèves

P137. Le nouveau programme ne répond pas efficacement aux besoins des apprenants

P138. Pour que le programme soit à la portée de tout le monde il faut qu'il réponde à ces conditions : la globalité, faisabilité. Il doit être accessible aux niveaux des élèves

$\mathrm{M}^{2} 114$. La réforme, les programmes... ne répondent pas aux besoins de nos apprenants

M141. Le niveau de l'élève ne s'adapte pas avec le nouveau programme

M265. Le contenu des manuels doit être plus attrayant pour l'apprenant et doit susciter sa curiosité et son implication

$\mathrm{L}^{3} 241$. Le manuel sans vie qui ne motive pas (il manque de tout: organisation, couleurs, informations, textes adaptés aux apprenants...)

L229. Le programme ne travaille pas les objectifs communicatifs de l'apprenant

L264. Les activités ne répondent pas aux besoins des apprenants

L285. Il faut choisir des sujets d'actualité et qui s'adaptent aux nouvelles générations afin de les motiver et de les mettre dans le bain

L312. Moi je ne travaille pas avec le manuel. Je cherche des textes d'actualité qui motivent

L313. Il faut prendre en considération les supports qu'on doit changer et remplacer par des sujets actuels les plus motivants

Plusieurs enseignants ont par ailleurs souligné le manque de prise en compte des caractéristiques de l'apprenant algérien d'une manière générale ou des spécificités régionales :

P.59. [on ne prend pas en considération] la représentation négative du français chez certains

P.64. Les personnes qui ont élaboré ces nouveaux programmes se sont basées sur un échantillon d'élèves et d'établissements, leurs visions étaient très restreintes

P153. J'aimerais préciser de par mon humble constat que les contenus des manuels scolaires ne véhiculent pas les valeurs algériennes et ne prennent pas en 
considération l'environnement direct des apprenants (un enfant du sud ne vit pas dans les mêmes conditions que celles d'un enfant du nord...) ce détail de représentions joue un mauvais tour quant à l'apprentissage des langues et constitue un véritable obstacle et pour les profs et pour les apprenants

P288. Le programme actuel n'est pas applicable avec les élèves de la wilaya d'Illizi en raison de leurs difficultés en matière de langue (français)

P.327. Les régions rurales ont des difficultés

M61. Les programmes en français ne sont pas adaptés à tous les apprenants des différentes régions de notre pays. En effet, les régions d'Algérie sont tellement hétérogènes, les cultures varient d'une wilaya à une autre, les programmes conçus pour la capitale par exemple ne sont pas adaptés au Sud ou à L'Est du pays et encore moins aux régions reculées et petits villages où les apprenants n'ont jamais entendu un seul mot de français

M67. (...) C'est un manuel [1AM] pour une certaine catégorie d'apprenants ceux des grandes agglomérations... donc pas tout le monde ! Malheureusement

L228. Il doit y avoir des programmes adaptés au niveau réel de l'élève algérien et qui prend en considération les différentes réalités socio-économiques de l'Algérie L315. Je pense que l'éducation d'une façon générale demande une réforme non seulement des moyens didactiques mais aussi on doit concevoir des savoirs selon les régions.

Plusieurs études (cf. Ammouden, 2017; Hamadache et Ammouden, 2017 ; Ghersa et Ammouden, 2018) et l'examen de plusieurs manuels utilisés actuellement permet de confirmer la plupart de ce que déclarent ces enseignants. Le fait que le MEN ne prévoit qu'un seul programme et qu'un seul manuel pour toutes les écoles de la très vaste Algérie suffit pour montrer que les profils des apprenants ne sont pas pris en considération. Le manque flagrant de textes d'auteurs algériens dans certains manuels (Hamadache et Ammouden, 2017), le manque d'authenticité et sens des contenus proposés (Ammouden, 2017) a déjà été signalé à plusieurs reprises.

On ne peut par ailleurs ne pas constater que de nombreux thèmes, textes et situations ne semblent pas de nature à motiver les apprenants ( $3 \mathrm{AM}:$ Je résume un récit historique portant sur un moment de l'Histoire nationale); 3 AS: Introduire un témoignage dans un fait d'Histoire). Quelques thématiques sont particulièrement malchoisies : c'est le cas des textes des séquences portant sur le fait divers (3 AM et 1 AS) qui se focalisent sur le vol, l'agression, le détournement, etc. Or, on pouvait enseigner le même genre de discours en s'appuyant sur des textes traitant de catastrophes naturelles, d'accidents, etc.

\section{La centration sur l'apprentissage?}

L'APC implique qu'on privilégie l'apprentissage fondé sur l'activité des apprenants aux dépens des pratiques transmissives. Les propos de plusieurs enseignants indiquent que la réforme a totalement échoué sur ce plan :

P168. L'approche par les compétences a-t-elle lieu vraiment... comment! ? Où est le document authentique, où est l'interaction avec le professionnel, où est ce prof qui fait parler les apprenants et qui sait guider, où est la situation problème... Où est la nécessité de changer le nom d'une approche alors que c'est l'ancienne qui circule toujours

P206. Beaucoup d'enseignants (surtout les nouveaux) ont un problème à enseigner avec l'approche par compétences et d'impliquer l'élève à la construction du savoir et de le mettre au centre d'apprentissage, et la majorité d'entre eux travaillent avec l'ancienne méthode 
M52. [L'l'APC] n'est pas appliquée en classe jusqu'à présent par un nombre important d'enseignants qui continuent à dispenser à leurs apprenants l'enseignement traditionnel

M80. Le problème qui se pose c'est que l'apprenant ne fournit pas d'efforts pour favoriser l'apprentissage. Dans ce cas, le professeur est le seul élément qui cherche l'information et la fait acquérir aux apprenants, car nos apprenants sont passifs ils attendent l'information pour la parcœuriser puis la reproduire lors des examens M129. La réforme n'a rien apporté de nouveau M141. L'enseignant reste toujours attaché à l'ancienne méthode

M268. C'est beau de parler sur l'approche par les compétences mais malheureusement sur le terrain on évalue les contenus et pas les compétences L21. La réforme élaborée, ces dernières années, d'abord, elle n'a pas apporté de changement

L84. Pour l'essentiel, la réforme n'a pas apporté du nouveau : elle n'a modifié ni les manières d'enseigner des enseignants, ni la manière d'apprendre des élèves

L229. Je crois que l'approche par les compétences n'est pas concrètement appliquée dans l'enseignement/apprentissage du FLE

L304. Concernant la réforme personne n'applique l'approche par les compétences car les enseignants ne connaissent pas le sens exact de l'approche par les compétences

L307. On fait du monologue dans les classes et ce n'est pas le cas de ma région seulement puisque je suis en contact avec des collègues d'autres wilayas qui se plaignent du même problème

L'examen des manuels confirme largement ces propos (cf. Ammouden, 2017). Les encadrés théoriques occupent dans le manuel de 1 AM (2016) jusqu'à la moitié de la première page de la leçon. Dans celui de la 2 AS (2013), on opte tout simplement pour la logique traditionnelle: théorie suivie d'exercices d'application. L'observation des pratiques montrent aussi que la logique de la transmission/mémorisation/restitution domine largement surtout au Primaire et au Moyen. Dans le secondaire, bien que les concepteurs de manuels évitent souvent cette logique, il arrive fréquemment que les enseignants prévoient des cours théoriques avant de passer aux exercices proposés dans le manuel. Si l'on prend l'exemple de l'enseignement de la grammaire, même si c'est la démarche inductive qui est recommandée dans les documents théoriques du MEN, elle n'est pas adoptée dans les manuels. Même quand les contenus théoriques sont présentés après les exercices de découvertes, force est de constater que ceux-là ne suffisent guère à découvrir toute la théorie qui les précède (cf. Ammouden, 2017; Ghersa et Ammouden, 2019).

Pour favoriser l'apprentissage, les activités doivent s'inscrire dans la Zone de Développement Proximale de l'apprenant: elles doivent donc être "suffisamment difficiles pour qu'il apprenne quelque chose, et suffisamment faciles pour qu'il ne se décourage pas » (Coïaniz, 1998). Or, il semble que les contenus proposés se situent largement audelà de cette zone. Les programmes correspondent-ils aux niveaux des apprenants ? L'écrasante majorité des enseignants répond par « un peu » (44\%) ou « non » $(46 \%)$.

Qu'en est-il des manuels? Le dépouillement des réponses donne globalement les mêmes pourcentages : « un peu » $(42 \%)$, « non » $(48 \%)$.

Les productions visées par les projets proposés dans les manuels sont-elles réalisables par les apprenants ? Si $27 \%$ répondent par l'affirmative, $52 \%$ pensent qu'ils ne sont pas vraiment à la portée des apprenants et $21 \%$ optent pour « non ». 
Les activités des manuels peuvent-elles être réalisées par les apprenants sans l'aide de l'enseignant ? $68 \%$ optent pour "non », $20 \%$ pour « un peu», tandis que ceux qui disent « oui » ne représentent que $12 \%$.

Quelques-uns des propos de nos enquêtés confirment les réponses ci-dessus :

P85. Les contenus sont trop difficiles pour les élèves. Les consignes sont mal conçues

P262. Les programmes proposés dépassent le niveau des apprenants

P270. Il est au-dessus des capacités des élèves. On s'est préoccupé des compétences à installer et on a raté la base de l'enseignement /apprentissage surtout au cycle primaire qui est lire écrire et compter

P273. Les nouveaux manuels de la deuxième génération ne sont pas à la portée des apprenants

P294. Au primaire, il vaut mieux favoriser l'apprentissage des notions de base et éviter le compliqué

M44. La réforme a diminué l'amour de l'école chez les apprenants. Car elle exige beaucoup de préparation dès le jeune âge

M67. Le programme de 1 année moyenne n'est pas à la portée de nos apprenants : textes supports difficiles à assimiler

M166. Cette approche par compétence et ces nouveaux programmes ne sont pas adaptés au niveau de nos apprenants

M.198. Les leçons proposées ne sont plus au niveau des apprenants

M 200. Les contenus des manuels ne correspondent pas au niveau réel de nos apprenants

L38. Les manuels sont mal conçus au niveau de la zone proximale de développement de l'apprenant et aux besoins de ces derniers

L. 81. Le contenu des programmes qui ne répond pas à la demande et qui n'est pas du tout adapté au niveau des apprenants

L. 83. Les contenus ne correspondent ni au niveau des apprenants (trop difficiles) ni à leur intérêt (ils sont ennuyeux)

L190. Il faut proposer des textes et des exercices qui sont à la portée des apprenants L313. Je trouve que les programmes actuels ne répondent ni aux niveaux ni aux compétences de nos apprenants

Par ailleurs, si l'une des exigences de l'APC est l'allègement des programmes, $13 \%$ uniquement de nos enquêtés pensent que les programmes correspondent aux volumes horaires alloués : $69 \%$ de nos enquêtés (dont $81 \%$ des PEP interrogés) répondent par non et $18 \%$ par « un peu» :

P48. Les programmes trop chargés

P217. Le programme de cette année est trop chargé surtout pour les élèves du primaire

P.233. Les élèves ont en marre de ce programme chargé : ils n'ont même pas le temps de respirer entre les séances

P242. Programme chargé surtout en 4 AP

P296. Le programme est trop chargé par rapport à l'ancien surtout au niveau des points de langue

M.201 Programme long pour tous les niveaux ; le volume horaire n'est pas adéquat

M129. La surcharge envahit toutes les matières et l'apprenant se trouve face à la difficulté d'assurer son année et maîtriser le tout

M211. Programme trop chargé et peu d'heures pour tout expliquer

L38. Nous demandons qu'il y ait également un volume horaire adéquat pour la réalisation de ces projets pédagogiques

L83. Les programmes sont surchargés

L97. Trop chargé le programme 
L220. Le programme est tellement surchargé qu'on n'a pas le temps de penser à enseigner correctement mais uniquement à avancer dans le programme, que les apprenants aient compris ou pas

L320. Les programmes sont trop surchargés et les volumes horaires alloués pour leur réalisation s'avèrent alors très insuffisants. Quand on ajoute à cela les nombreuses grèves des enseignants (...) il est impossible de réaliser le programme correctement. On est obligé de faire vite pour terminer ; conséquence : on bricole, on fait semblant ...mais c'est surtout impossible de s'offrir le luxe de penser au développement des compétences dans ces conditions

Cette surcharge des programmes (mais surtout la difficulté des contenus des manuels) conduit les enseignants à donner quotidiennement plusieurs travaux à faire à la maison, pour terminer le programme. Or, les contenus ne sont pas à la portée des élèves du Primaire notamment. Cette situation a obligé les parents à adhérer massivement aux nombreux groupes des enseignants sur Facebook et à demander des explications et orientations pour aider leurs enfants à solutionner les exercices remis, voire souvent pour les faire eux-mêmes, avouent certains. Il arrive souvent que quelques-uns dénoncent la difficulté des exercices, le flou des consignes, et demandent de l'aide, etc. Cela a enfin obligé de nombreux parents à payer des cours de soutien à leurs enfants même si ces derniers ne sont pas en classe d'examen (BEM, BAC), les seuls - et pas nombreux d'ailleurs - qui avaient besoin de recourir à ces cours il y a quelques années. Ces cours concernent maintenant un nombre de plus en plus important d'apprenants et atteignent même ceux des écoles primaires.

\section{L'amélioration des programmes et des manuels ?}

La réforme devait surtout se traduire par l'amélioration des programmes et des manuels. Les enseignants pensent que les deux sont mal conçus :

P298. Programme nul

P289. Les programmes ne sont pas bien conçus, ne motivent pas les élèves, la majorité des élèves trouvent des difficultés à apprendre le FLE

P298. Manuel mal organisé n'aide pas ni l'enseignant ni l'apprenant ni le parent d'élève

M165. Juste de nouveaux manuels avec une nouvelle couverture rien d'autre n'a changé

M303. Je pense que les concepteurs des manuels viennent d'une autre planète

L36. Les manuels proposés aux apprenants ne répondent pas aux fondements de l'APC

L38. Les manuels sont mal conçus

L134. Il faut actualiser les textes des manuels en choisissant des textes d'actualité pour chaque actualisation

L178. Il est nécessaire de revoir le programme du secondaire. Les manuels sont mal conçus

L216. Des programmes à améliorer

L220. Les programmes et les manuels ne correspondent pas à ce à quoi on s'attendrait.

L230. Il reste beaucoup à faire notamment pour les manuels du français au secondaire

L263. Les manuels scolaires ne répondent pas du tout aux programmes

L264. Le manuel scolaire ne contient pas les compétences ni les 4 aptitudes

L287. Il est nécessaire de proposer des manuels qui respectent l'approche par les compétences

L314. À prévoir d'autres programmes plus motivants, réalisables durant l'année 
Ils sont également plusieurs à nous confier que les manuels comportent de nombreuses erreurs :

P48. Les manuels sont pleins de fautes qu'elles soient d'ordre logique ou orthographique. On prend conscience que ça n'a pas été fait avec sérieux tout simplement, si ça a été relu au moins une fois par un groupe élargi, ils auraient découvert d'une manière consensuelle les erreurs, des millions d'Algériens payent le non sérieux d'un groupe limité d'individus incompétents

P133. Les manuels sont des copier-coller pleins de fautes et incorrections

M100. Les livres sont bourrés de fautes - orthographique, syntaxiques, typographiques, et j'en passe. (...) Les erreurs et autres incorrections sont dues à la précipitation avec laquelle ils ont élaborés. 4 mois pour préparer un manuel scolaire est hors norme. Les normes internationales exigent 18 mois, plus son expérimentation dans des écoles pilotes. Chose qui n'existe plus depuis l'école fondamentale.

L83. On sent que les manuels sont à chaque fois conçus dans l'urgence en témoignent les nombreuses erreurs qu'on y trouve

Notons à ce sujet que plusieurs études ont montré que les nouveaux programmes et manuels s'avèrent même parfois moins corrects et moins rentables, sur le plan didactique, que ceux qu'ils ont remplacés (cf. Saidoun et Ferroukhi, 2017).

\section{Les projets-élèves ?}

31 Comme nous l'avons déjà montré (Ammouden, 2017), nous avons constaté une grande confusion entre la notion de projet-élèves (désigné dans les documents du ministère par projet pédagogique) et le projet didactique. Si les définitions citées dans le RGP et le GMEP correspondent à la théorie de la pédagogie du projet et à celle du projet-élève (Huber, 2005), ce n'est pas le cas de celles données dans plusieurs programmes et documents d'accompagnement. On explique par exemple dans les documents d'accompagnements des programmes de la 1 AS $(2005: 3)$ et de la 2 AS (2006: 03) :

Le projet considéré dans sa globalité, constitue l'organisateur didactique d'un ensemble d'activités. Il est organisé en séquences, ayant une cohérence interne et des intentions pédagogiques. Le projet permet d'installer une ou plusieurs compétences définies dans le programme. Il permet aussi de passer d'une logique d'enseignement à une logique d'apprentissage

En entreprenant une démarche de projet le professeur accepte :

de tenir compte des besoins et des intérêts des apprenants,

de négocier avec les apprenants les objectifs et les moyens,

d'agir comme médiateur et non comme dispensateur de savoir

L'apprenant impliqué dans un projet est un partenaire actif dans le processus de son apprentissage. Il "apprend à apprendre " par une recherche personnelle. (MEN, 2005, 2006).

Si les trois premières phrases de cette définition traitent du projet didactique, les autres (à partir de «Il permet aussi de passer ») renvoient clairement à la logique du projet-élèves.

On peut repérer la même confusion dans le passage ci-après qu'on trouve dans plusieurs documents, dont le document d'accompagnement au programme de la 5 AP (2009:18) et le programme de français de la 1 AM (2013:10). On constate que le premier paragraphe traite du projet-élèves, alors que le deuxième concerne le projet didactique :

Le projet pédagogique n'est pas une fin (but) en soi, c'est un moyen pour confronter les apprenants à des obstacles et provoquer des situations d'apprentissage 
significatives. Il ne doit en aucun cas occulter les apprentissages au profit du produit à réaliser. C'est donc le cadre dans lequel se réalisent les apprentissages.

Le projet considéré dans sa globalité, constitue l'organisateur didactique d'un ensemble d'activités. Il est conçu sous forme de séquences ayant une cohérence interne et des intentions pédagogiques. Le projet permet d'installer une ou plusieurs compétences définies dans le programme

explique que les apprentissages linguistiques doivent être mis au service de la réalisation du projet qui est "une situation complexe susceptible d'intégrer plusieurs compétences et de mobiliser des connaissances diverses » (p. 17), on ne traite juste après des contenus qui concernent les séquences didactiques. La confusion suggérée ici implicitement sera amplifiée par l'énumération qui se trouve à la fin de la partie censée contenir des exemples de projets suggérés. On y trouve des productions assez exigeantes et intéressantes telles que "un album de lecture » et " un spectacle », mais aussi d'autres telles qu'une fiche technique, une lettre, une carte de vœux, un poème ( $\mathrm{p}$. 32), qui conviendraient dans le meilleur des cas à des production à associer à des séquences didactiques.

le projet-élèves est au cœur de l'APC, il est clair que les confusions véhiculées par les outils didactiques font qu'on parle de pédagogie du projet mais que celle-ci est en réalité réduite à des projets didactiques. Le fait que les intitulés des projets, très télécommandés et ne laissant guère de place au choix et à la négociation des apprenants (Ammouden, 2017), soient donnés dans les programmes et manuels suffit pour confirmer qu'on parle de projet-didactiques.

Les projets-élèves proposés sont-ils bien choisis? Si $21 \%$ de nos enquêtés répondent par oui, la plupart optent pour les réponses « un peu» $(44 \%)$ ou «non» $(35 \%)$. Les réponses des PES, s'avèrent moins positives : « un peu » $(46 \%)$, « non » (44 \%). Ajoutons qu'il est fort possible que la confusion dont nous venons de traiter ait légitimement conduit les enseignants, comme les concepteurs de manuels, à confondre eux aussi entre les deux. En effet, la réalité révèle que les projets-apprenants proposés sont souvent de simples projets d'écriture, trop scolaires, trop disciplinaires (Ammouden, 2017) et trop individuels, surtout dans les pratiques, en témoignent les exemples suivants :

A la fin de ce projet, tu seras capable de comprendre et de produire un texte argumentatif qui s'appuie sur l'explication.

A la fin de ce projet, tu seras capable de comprendre et de produire un texte argumentatif qui s'appuie sur la narration

A la fin de ce projet, tu seras capable de comprendre et de produire un texte argumentatif qui s'appuie sur la description. (4 AM)

Lire et écrire un texte documentaire (5 AP)

Afin de célébrer les journées de la propreté et de l'alimentation qui se déroulent les 15 et 16 octobre de Rédiger une nouvelle fantastique (3 AS).

Nous pensons que le caractère trop scolaire de ses projets, qui sont en réalité de simples activités scripturales (comprendre/lire un texte), est dû aux confusions dont nous traitons plus haut et au fait que ni la logique de projet-didactique, ni celle de projet-élèves; ni celle de la compétence ne semblent être suffisamment assimilées par les concepteurs des programmes et des manuels. 


\section{L'entrée par les compétences ?} des intitulés de projets :

« Nous rédigeons un recueil de contes qui sera lu aux camarades d'un autre collège Dans le cadre du concours de lecture, mes camarades et moi interprétons nos fables Nous rédigeons un recueil de légendes à présenter le jour de la remise des prix » (2 AM)

« Réaliser une campagne d'information à l'intention des élèves du lycée.

Rédiger une lettre ouverte à une autorité compétente pour la sensibiliser à un problème et lui proposer des solutions.

Écrire une petite biographie romancée ». (1 AS)

«Dans le cadre de la commémoration d'une journée historique, réaliser une recherche documentaire puis faire la synthèse de l'information à mettre à la disposition des élèves dans la bibliothèque de l'établissement

Organiser un débat d'idées puis en faire un compte-rendu.

Dans le cadre d'une journée " portes ouvertes », exposer des panneaux sur lesquels seront reportés des appels afin de mobiliser les apprenants et les visiteurs autour de causes humanitaires » (3 AS).

Si l'on peut donc certes parler d'une certaine ressemblance entre ces intitulés de projets et l'énoncé de la compétence proposé par Miled, force est d'admettre qu'ils présentent de nombreuses insuffisances. Ces énoncés, renvoyant à des projet-élèves ne doivent pas être disciplinaires ; ils sont utilisés comme intitulés de projets didactiques ; les situations sont trop télécommandées et laissent peu de place à la négociation des apprenants; les compétences désignées sont trop liées à une situation bien précise et donc peu transférables; ils se focalisent trop sur l'environnement scolaire, ce qui ne favorise ni le développement des compétences, ni leur transfert dans l'environnement extrascolaire (Ammouden, 2017).

Il faut par ailleurs mentionner qu'à partir de ces intitulés, plus ou moins corrects, on retombe dans une entrée par contenus linguistiques dont les énoncés ne correspondent guère aux objectifs spécifiques ou ressources permettant le développement des compétences, comme le montrent les intitulés des séquences du $1^{\mathrm{er}}$ projet de la $1 \mathrm{AM}$ (2016) ou de celles du manuel de 1 AS:

J'explique l'importance de se laver correctement; J'explique l'importance de manger convenablement ; J'explique l'importance de bouger régulièrement (1 AM)

Contracter des textes; Résumé à partir d'un plan détaillé ; Résumé en fonction d'une intention de communication (1 AS)

Nous pouvons donc conclure que les entrées n'obéissent pas vraiment à la logique des compétences : c'est toujours l'entrée par contenus, voire par thèmes, qui domine. 


\section{Approche globale de la langue, enseignement par séquences didactique et par genres de discours} est encouragée par la pédagogie par objectifs (PPO), préconisent l'approche globale des phénomènes de langues (Ammouden, 2017). Ils privilégient une entrée par tâches Miled (2011). Cela devrait conduire à bannir les entrées grammaticales, lexicales, thématiques, etc., au profit d'entrées discursives. Le dispositif de la séquence didactique (Dolz-Mestre, Noverraz et Schneuwly, 2002) convient très bien à cette logique, pourvu que leurs concepteurs délaissent la logique des types de textes, qui constitue "plus un obstacle méthodologique ", au profit de la logique des genres qui s'est avérée plus correcte, plus pertinente (Adam, 2005).

entant, l'examen des tables de matières des manuels utilisés actuellement permet de constater clairement que ce sont toujours les conceptions traditionnelles, dont notamment celles des actes de paroles et des types de textes, qui y dominent. Ces conceptions sont d'ailleurs explicitement préconisées dans les programmes, guides et documents d'accompagnements.

Les séquences portent parfois sur des "situations" ou des "thèmes " auxquels sont associés des actes de paroles :

Ma nouvelle école (actes de paroles: Présenter/Présenter: Présenter/ Saluer/ prendre congé);

Mes camarades de classe (actes de paroles : Interroger /Exprimer une préférence);

Mon métier d'élève (3AP)

Tout en s'inscrivant dans la logique des actes de paroles, on retient en 4 AP et 5 AP des objectifs liés à un projet :

Repérer des événements importants; Présenter des paroles des personnages dans un dialogue ; Repérer les paroles des personnages ; Repérer la ponctuation dans une comptine, un poème (4AP)

Identifier le thème d'un texte documentaire ; Repérer les informations essentielles dans un texte documentaire ; Retrouver un processus de fabrication (5AP)

On privilégie dans le nouveau manuel de 1 AM (2016), l'entrée par types de textes, inscrite dans une optique thématique. Les intitulés des séquences didactiques indiquent qu'il n'y souvent que le thème qui change et donc que la place accordée aux approches thématiques et par « situations ", remises en question pourtant depuis déjà longtemps, est toujours très importante dans les manuels actuels :

Projet 1 : J'explique l'importance de se laver correctement; J'explique l'importance de manger convenablement ; J'explique l'importance de bouger régulièrement.

Projet 2 : J'explique les progrès de la science; J'explique les différentes pollutions ; J'explique le dérèglement du climat. d'expression écrite. C'est le cas en 3 AM (Je résume un récit historique portant sur un moment de l'Histoire nationale); en 1 AS (Contracter des textes ; Résumer à partir d'un plan détaillé ; Résumer en fonction d'une intention de communication) et en 3 AS (Introduire un témoignage dans un fait d'Histoire).

Elles s'articulent enfin parfois sur des parties de genres de discours. C'est le cas en $3 \mathrm{AM}$ (J'insère le portrait de mon personnage/un autoportrait dans un récit de vie). C'est clairement le cas en 2 AM (« La situation initiale du conte»; «La suite d'événements 
dans le conte »; "Le portrait des personnages du conte "; "La situation finale du conte »). Nous pouvons dire dans ce cas que le projet didactique s'articule autour d'un genre, mais que ce n'est pas le cas des séquences.

Il est enfin à préciser, comme nous l'avons déjà souligné (Ammouden, 2017; Hamadache et Ammouden, 2017), que même quand que les intitulés indiquent que les séquences s'articuleraient autour de genres de discours (conte, nouvelle, fable, reportage, lettre, etc.), l'exploitation didactique proposée dans programmes et manuels obéissent à la théorie des types de textes. Amar Ammouden (2015) a déjà montré, à ce sujet, que le fait que des noms de genres de discours soient parfois utilisés dans les intitulés n'a pas eu d'impacts sur les pratiques des enseignants "faute de recommandations clairement formulées dans les manuels et les documents d'accompagnement des programmes » (p. 8).

Pour ce qui est de l'articulation des compétences travaillées et des contenus linguistiques, l'examen de tous les manuels révèle que c'est la logique de l'atomisation qui y domine. Les compétences de l'oral - quand elles sont prises en considération sont séparées de celles de l'écrit (cf. Ammouden, 2013, 2017). Les apprentissages linguistiques également sont souvent très morcelés. Cela est particulièrement flagrant dans les manuels du Primaire et du Moyen, dans lesquels les contenus sont répartis sur de nombreuses rubriques :

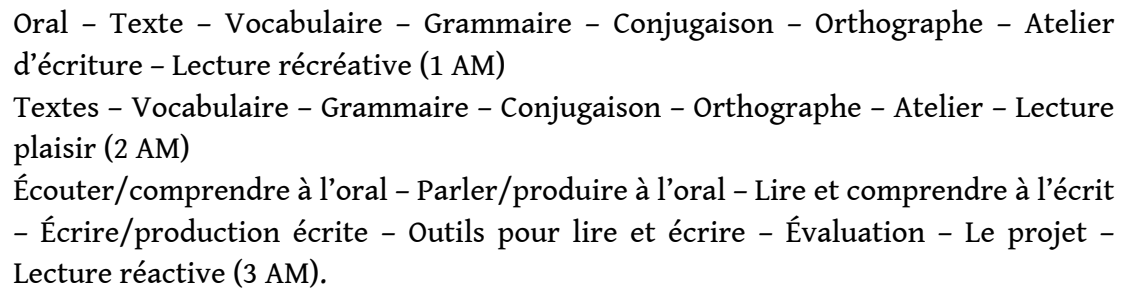

Les tables des matières des manuels indiquent que leurs concepteurs semblent avoir du mal à se détacher du morcèlement et de l'atomisation des contenus prônés par la PPO et des théories des actes de parole et des types de textes qui ont caractérisé les approches communicatives. La théorie de l'APC implique pourtant l'approche globale et intégrée des phénomènes de la langue, unique moyen par lequel les compétences peuvent être développées et par lequel on peut divorcer avec la logique de la simple transmission de connaissances purement linguistiques au profit du travail de compétences discursives de haut niveau. Cela pouvait être favorisé par l'enseignement par projets et séquences didactiques, recommandé pourtant dans les documents institutionnels algériens, pourvu que les concepteurs des programmes et manuels aient respectés les fondements théoriques de ces dispositifs, ce qui n'est guère le cas actuellement.

\section{Les situations-problèmes et situation d'intégration ?}

Si ce qu'on explique, dans plusieurs documents théoriques du MEN, au sujet des situations-problèmes ou d'intégration est globalement correct, on repère à plusieurs reprises des indications et propositions qui sont très peu ou pas du tout en conformité avec la théorie. Celles-ci représentent dans les meilleurs des cas de simples activités.

53 C'est le cas des situations d'intégration ou des objectifs d'intégration suggérés dans le Curriculum de la 2AS (2006:26). On y explique la situation permet d'intégrer les acquis 
"pour résoudre une situation-problème", avant de proposer de simples activités d'écriture

Ex 1 : Vous avez un correspondant habitant un autre pays. Rédigez un texte pour lui faire connaître votre région et l'inciter à venir la découvrir

Ex 2: Votre ville ou votre quartier croule sous les ordures. Rédigez untexte s'adressant à vos concitoyens pour les sensibiliser et développer leur espritcivique.

b - Situation d'intégration pour l'année (OIT)

Votre lycée organise une journée d'informations sur le sida. Vous écrivez un texte pour marquer votre participation à cet événement. Ce texte prendra la forme que vous jugerez la plus adéquate.

C'est aussi le cas des situations-problèmes suggérées, respectivement pour les classes de $1 \mathrm{AM}$ et de $2 \mathrm{AM}$, dans le document d'accompagnement du programme de français au cycle moyen (2016):

"À l'écoute de ton émission radiophonique préférée, tu entends un médecin expliquer les problèmes de santé que peut entrainer la "malbouffe ». Intéressé(e) par son exposé, tu décides de prendre des notes pour informer tes camarades sur les dangers de cette manière de se nourrir ".

«C'est la journée internationale du livre. Ta classe participe à un concours de lecture de contes qui se déroule dans ton école, devant les parents d'élèves. Vous devez sélectionner des contes et les mettre en voix ».

C'est également le cas de la suivante, proposée dans le manuel de la $1 \mathrm{AM}$ :

« (...) Vous devez montrer - au moyen d'un texte illustré- comment le vélo peut jouer un rôle important dans la vie des individus, notamment dans le maintien d'une bonne santé et la réduction des embouteillages qui augmentent le taux de pollution de l'air ».

Il semble que qu'on confonde entre situation-problème et mise en situation des activités. En effet, par exemple les activités proposées plus haut ne consistent en réalité qu'à pratiquer la technique de la prise de note à partir d'un oral, à oraliser un conte écrit, à écrire un «texte argumentatif » au sujet du vélo; or, ce qu'on attend d'une situation-problème ou d'intégration dépasse largement ces objectifs.

\section{La formation des enseignants, des inspecteurs, des concepteurs?}

Si la réforme a été qualifiée de globale, c'est entre autres parce qu'elle devait concerner aussi la formation des principaux acteurs de l'éducation. Est-ce le cas?

Les enseignants ont-ils bien compris les implications de l'approche par les compétences? $17 \%$ uniquement répondent par l'affirmative. La plupart des enseignants des trois paliers - avec des pourcentages très proches - pensent qu'ils ont peu $(52 \%)$ ou pas du tout ( $31 \%)$ compris les principes cette approche. Certains nous confient :

P50. Le problème vient du niveau des enseignants qui atterrissent sur les estrades sans pré recuis pédagogique

P.117. ça reste que des théories apprises et non acquises à l'université et dont la pratique est absente dans les établissements par manque de formation

P164. La réforme qu'a connue l'école algérienne est vouée à l'échec (...) en omettant la pièce angulaire du système éducatif en entier qui est l'enseignant. (...) Son droit de recevoir une formation continue adéquate, une formation qui va l'épauler, l'outiller le conforter. Une formation articulée entre théorie et pratique 
M20. Proposer des formations pour les enseignants afin de leur permettre de bien comprendre le concept "approche par compétence"

M32. Il faut former les enseignants pour la réussite de cette réforme ambitieuse

$M$ 125. Beaucoup de professeurs jusqu'à maintenant n'arrivent pas à comprendre c'est quoi l'approche par compétence (...) on demande plus d'éclairages et de formations pour que les professeurs saisissent mieux

M 128. La réforme de 2ème génération est l'actualité actuellement. Il faut bien se former pour bien mener l'enseignement/apprentissage

M 198. Je trouve que le professeur est obligé de suivre un programme sans formation et sans explications il doit appliquer le programme et c'est tout

L36. Les professeurs manquent de formation. Il faut qu'il y ait des ponts entre l'enseignement supérieur et l'éducation nationale

L51. Il y a des profs eux-mêmes n'ont pas compris l'approche par compétence

L119. Je pense que la réforme des programmes doit indéniablement passer par la formation des praticiens de l'éducation. On peut ramener les meilleurs programmes au monde mais leur efficacité demeure nulle si ceux qui sont chargés de les appliquer ne sont pas formés en conséquence et ne possèdent pas les compétences qu'il faut

D'autres enseignants expliquent que les inspecteurs eux-mêmes n'ont pas compris cette approche

P149. L'approche par compétence n'est pas encore acquise par les inspecteurs ni par les enseignants

P161. La réforme des programmes a créé beaucoup de questions car les inspecteurs de la matière eux même sont dans le sombre

p185. Toute réforme doit être encadrée: formation des enseignants voire des inspecteurs

P300. Parfois je pose des questions aux inspecteurs et ils ne me répondent pas. Ils n'ont pas encore trouvé des solutions pour quelques problèmes que les enseignants ont signalés

M 125. (...) ces programmes de 2 ème génération même les inspecteurs n'ont rien compris

L147. L'absence d'une formation réelle sur l'APC. (...) à cause de l'incompétence du corps d'inspection qui est comme un agent administratif seulement

L234. Comment espérer parvenir à une reforme (...) quand le terme "compétences" lui-même fait défaut (notamment en parlant des inspecteurs et des enseignant)

D'autres évoquent encore le manque de formation des concepteurs de programmes et de manuels. Un PEP estime que «Les concepteurs des programmes manquent de formation sérieuse " (P139). Un PEM, sollicité pour l'évaluation d'un des manuels de $2^{\text {ème }}$ génération de la réforme explique :

M100. Les manuels sont conçus par des inspectrices en collaboration avec quelques enseignants lesquels n'ont aucune notion sur l'évaluation du manuel scolaire. (...)Je vous en parle, car j'ai travaillé avec les inspectrices qui les ont conçus. (...) Ce que je ne comprends pas, pourquoi on sollicite des inspectrices pour le manuel alors que ce n'est pas leur "métier", c'est-à-dire leur profil. Il y a les concepteurs de méthodes, les concepteurs de programmes, les concepteurs de manuels, des inspectrices pour accompagner les enseignants dans leurs opérations de formation et veiller à ce que les programmes soient appliqués. On a envoyé il y a quelques années des gens du domaine pour une formation de 6 mois au Canada spécialement pour le manuel scolaire. Une fois de retour, ils ont été écartés, et on a confié cette tâche à des inspectrices car elles font partie du sérail(...).

61 Si la formation des acteurs concernés par la réforme a été prévue, il semble que l'objectif n'ait pas été réalisé. 


\section{Les conséquences?}

\section{sont-ils réalisés?}

Pour toutes les insuffisances évoquées plus haut, les enseignants sont persuadés que la réforme a été très mal gérée sur le terrain, qu'elle leur a été imposée, que personne n'a demandé leur avis, qu'on ne les a pas suffisamment formés pour travailler avec cette approche, que ceux à qui on a confié l'élaboration des nouveaux programmes et manuels ont «bâclé » le travail, etc. Ils considèrent que c'est un échec et ils ont décidé de ne pas l'appliquer:

P117. la réforme et le nouveau programme (tellement on a vu de programmes) la plupart des enseignants ne suivent pas le programme et (veuillez excuser l'expression) ils ne font qu'à leurs têtes

P146. Quand le programme sera réalisé et révisé et en plus choisi par les enseignants qui sont au domaine ...on récolte le bonne fruit. Sinon le résultat sera toujours négatif

P279. Le programme est à refaire avec la contribution des enseignants

P292. Enseignants et élèves sont devenus des cobayes pour ceux qui imposent ces réformes à notre système éducatif !

C171. Vive le système traditionnel

L287. Je voudrais aussi qu'on permette aux professeurs de participer à la réalisation des programmes et des manuels

L326. à notre avis, ce sont les enseignants qui durcissent et freinent le projet de la réforme ce durcissement se traduit souvent par un maintien des pratiques traditionnelles voire une application erronée du programme

Dans ces conditions, il faut s'attendre à ce que les chances que les objectifs de la réforme soient atteints s'avèrent très réduites.

La réforme visait surtout le développement de compétences transférables. A-t-on concrétisé cela? A l'exception de $23 \%$ de nos enquêtés qui répondent par oui, les autres estiment que dans les pratiques cet objectif n'est que peu (46\%) ou pas du tout (31\%) favorisé.

La réforme a-t-elle contribué à améliorer le niveau des apprenants? La plupart des enseignants $(82 \%)$ répondent par «un peu» $(36 \%)$ ou par «non» $(46 \%)$. Si le pourcentage des PEM qui répondent par « oui » représente $24 \%$, ceux des PEM et des PES diminuent respectivement à $15 \%$ et à $14 \%$. Si les PEP qui répondent par «non » représentent $36 \%$, la même réponse est donnée par $49 \%$ des PEM et $56 \%$ des PES.

De nombreux enseignants pensent ainsi que la réforme a échoué, comme l'illustrent ces propos:

P292. Ces réformes sont comme quelqu'un qui avance d'un pas et qui recule de deux pas! Ces nouveaux programmes ont embrouillé l'équipe pédagogique ainsi que les apprenants!

C172. On donne du pain à celui qui meurt de soif

L22. Je pense que la réforme (éducative, mise en place à partir de 2003, n'a pas abouti aux résultats attendus)

L83. La réforme a été lancée sans que les enseignants ne soient informés ni consultés. (...). En ce qui me concerne la réforme est un échec.

L110. Tout est à refaire!

L218. Une réforme qui laisse beaucoup à désirer

L224. C'est une théorie utopique.

L239. Cette réforme est à revoir dans son intégralité

L253. Un désastre sur tous les niveaux 
L278. Les nouveaux programmes, censés améliorer le rendement scolaires de nos enfants en les amenant à réfléchir par eux-mêmes et à développer leur esprit critique, ne peuvent réussir (...)

L297. Je trouve que l'ancien programme avec la nouvelle approche ça pouvait mieux marcher avec nos apprenants c'était beaucoup plus organisé et suivait une certaine logique.

$\mathrm{Au}$ terme de cette étude, nous pouvons conclure que si ce qui est recommandé théoriquement dans le RGP et dans le GMEP est généralement conforme à la théorie de l'APC, au fur et à mesure qu'on se rapproche des pratiques des classes, on s'éloigne des fondements de cette approche. À titre d'exemple, on recommande dans les documents du MEN de favoriser l'apprentissage aux dépens de l'enseignement transmissif, de travailler par projet-apprenants, par séquences didactiques, par situations-problèmes, etc. Ce qu'on trouve dans les manuels ne correspond que très rarement à ces recommandations : ce sont toujours les conceptions traditionnelles qui prédominent. Qu'en est-il des pratiques des enseignants ? Les réponses et propos des 322 enseignants de français qui remplissent nos questionnaires ont permis la mise en en évidence de plusieurs insuffisances, erreurs et incohérences que l'examen des programmes, des documents d'accompagnements et des guides remis aux enseignants et l'observation des pratiques confirment largement. Les initiateurs de la réforme ont commis et commettent toujours, selon les enseignants, l'erreur de confier la conception des programmes et des manuels à des inspecteurs et enseignants insuffisamment formés. Ils auraient surtout commis l'erreur de n'avoir pas suffisamment informé/formé les enseignants et de n'avoir presque rien fait pour avoir leur adhésion à ce projet. Cela a fait que les enseignants sentent que cette réforme leur a été imposée et ils ont alors, volontairement ou involontairement, résisté au changement. C'est tout cela qui fait que les principes de l'APC, très présents dans le RGP et le GMEP, s'avèrent peu respectés dans les programmes, beaucoup moins dans les manuels et très ignorés dans les pratiques enseignantes. Cette étude confirme qu'il ne suffit pas qu'on décrète une réforme pour que celles-ci se réalise dans les pratiques (Martinez, 1996). Si, le MEN algérien a décrété une réforme globale et l'adoption de l'APC, les concepteurs des programmes et des manuels mais surtout les enseignants ne semblent guère être prêts à adopter les principes de cette approche. Il aurait été préférable d'associer ces acteurs à la réforme, de les préparer à s'adapter aux changements importants qu'elle implique avant de la décréter.

\section{BIBLIOGRAPHIE}

ADAM Jean-Michel, « La notion de typologie de textes en didactique du français : une notion "dépassée" ?», in. Recherches, 42, 2005, 11-23.

AIT DJIDA Mohand Amokrane, «L'approche par les compétences et la problématique du transfert », in. Synergies Algérie, 5, 2009, 55-63. 
AMmouden Amar, «Le français au lycée en Algérie : des " types " de textes aux "genres" de discours ", in. Multilinguales, $\mathrm{n}^{\circ}$ 6, 2015, consultable sur : http://journals.openedition.org/ multilinguales/976 (consulté le 05 juin 2018).

AMMOUDEN M'hand, « La place de quelques principes de l'Approche Par les Compétences dans les nouveaux manuels de français ", dans S. Saidoun et K. Ferroukhi (éds) Le manuel scolaire en Algérie à l'ère des réformes : enjeux et perspectives, Éditions universitaires européennes, Berne, 2017, 20-37.

AMMOUDEN M'hand, «Place de l'articulation oral/écrit dans l'enseignement du français dans le secondaire algérien », in. Synergies Mexique, 3, 2013, 31-44, disponible sur https://gerflint.fr/Base/ Mexique3/Ammouden.pdf

BEACCO, Jean-Claude, L'approche par compétences dans l'enseignement des langues : Enseigner à partir du Cadre européen commun de référence pour les langues, Didier, Paris, 2007.

BOUTIN Gérald, «L'approche par compétences en éducation : un amalgame paradigmatique », in. Connexions, 81, 2004, 25-41, disponible sur https://www.cairn.info/revue-connexions-2004-1page-25.html (Consulté le 5 juin 2018).

CARBONNEAU Michel et LEGENDRE Marie-Françoise, « Pistes pour une relecture du programme de formation et de ses référents conceptuels ", in. Vie pédagogique, 123, 2012, 12-17.

CHAUVIGNÉ Christian et COULET Jean-Claude, «L'approche par compétences : un nouveau paradigme pour la pédagogie universitaire ? », in. Revue française de pédagogie, 172, 2010, 15-28, disponible sur http://journals.openedition.org/ rfp/pdf/2169 (Consulté le 15 mai 2018).

CoÏANIZ Alain, « L'entrée dans le langage en français langue non maternelle », 1998, disponible sur http://guyane.scola.ac-paris.fr/ftp_public/Coianiz.pdf (Consulté le 5 juin 2018).

DOLZ-MESTRE, Joaquim., NOVERRAZ Michèle \& SCHNEUWLY Bernard (dirs), (2002), S'exprimer en français. Séquences didactiques pour l'oral et pour l'écrit, Volume 4, De Boeck/COROME, Bruxelles, 2002.

HUBER Michel, Conduire un projet-élèves, Paris, Hachette-Éducation, 2005.

GHERSA Assia ET AMMOUDEN M'hand, « Apports sociodidactiques pour l'enseignement du français dans le cadre de la didactique convergente : possibilités et obstacles ", in. Didacstyle, $\mathrm{n}^{\circ} 10$, p. 106-125, consultable sur https://www.asjp.cerist.dz/en/article/56017.

GHERSA Assia ET AMMOUDEN M'hand, « La place de l'enseignement inductif de la conjugaison du français dans le Moyen algérien », in. Expressions, n 8, 2019 (à paraitre).

HAMADACHE Tahar et AMMOUDEN M'hand, « Les textes littéraires dans l'enseignement du français en Algérie », in. Les Langues Modernes, Volume 111, n 4, 2017, 24-32.

HUBER Michel, Conduire un projet-élèves, Hachette, Paris, 2005.

MILED Mohamed, « Contextualiser l'élaboration d'un curriculum de français langue seconde : quelques fondements épistémologiques et méthodologiques ", dans P. Martinez, M. Miled et Tirvassen (coord), Curriculum, programmes et itinéraires en langues et cultures, Français dans le monde, $R \& A, 49,2011,64-75$.

PERRENOUD Philippe, « Des savoirs aux compétences : les incidences sur le métier d'enseignant et sur le métier d'élève », in. Pédagogie collégiale (Québec), V9-2, 1995, 6-10, disponible sur http:// www.unige.ch/fapse/SSE/teachers/perrenoud/php_main/php_1995/1995_09.rtf (Consulté le 5 juin 2018).

PERRENOUD Philippe, Construire des compétences dès l'école, 5éd., ESF, Issy-les-Moulineaux 2008. 
SAIDOUN Souad et FERROUKHI Karima (éds), Le manuel scolaire en Algérie à l'ère des réformes : enjeux et perspectives, Éditions universitaires européennes, Berne, 2017.

\section{NOTES}

1. La lettre « $P$ » qui précède les chiffres signifie que les enquêtés concernés sont des enseignants de l'école primaire.

2. La lettre « $M$ » qui précède les chiffres signifie que les enquêtés concernés sont des enseignants de l'enseignement moyen (Collège).

3. La lettre « $\mathrm{L}$ » qui précède les chiffres signifie que les enquêtés concernés sont des enseignants de l'enseignement secondaire (lycée).

\section{RÉSUMÉS}

Le Ministère de l'Éducation Nationale algérien a recommandé d'adopter l'approche par les compétences dès 2002. Dans quelle mesure les documents théoriques institutionnels et les manuels de langue française sont-ils en conformité avec les fondements de cette approche? Comment les enseignants reçoivent-ils et évaluent-ils la réforme ? Cette dernière a-t-elle eu les effets escomptés? L'étude montre que la réforme semble rester au stade des intentions, entre autres, parce que les programmes et manuels véhiculent de nombreuses insuffisances et parce que les enseignants, qui n'ont pas été préparés à celle-ci, la rejettent.

The Algerian Ministry of National Education recommended adopting the competency-based approach as early as 2002 . To what extent are the institutional theoretical documents and the French-language textbooks in conformity with the foundations of this approach? How do teachers receive and evaluate reform? Has the latter had the desired effects? The study shows that the reform seems to remain at the stage of intentions, among others, because the programs and textbooks convey many shortcomings and because the teachers, who have not been prepared for it, reject it.

\section{INDEX}

Mots-clés : Approche par les compétences, programmes, manuels, pratiques de classes, formation des enseignants

Keywords : Skills approach, programs, manuals, classroom practices, teacher training

\section{AUTEUR}

\section{M'HAND AMMOUDEN}

Laboratoire LAILEMM, Université de Bejaia, Algérie 